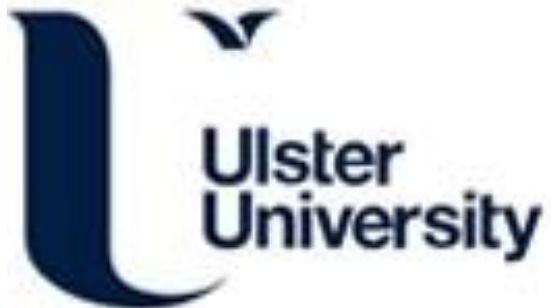

\section{Cannibalism and chaos in the classroom}

Abernethy, G. M., \& McCartney, M. (2017). Cannibalism and chaos in the classroom. International Journal of Mathematical Education in Science and Technology, 48(1), 117-129.

https://doi.org/10.1080/0020739X.2016.1184337

Link to publication record in Ulster University Research Portal

\section{Published in:}

International Journal of Mathematical Education in Science and Technology

Publication Status:

Published (in print/issue): 07/01/2017

DOI:

10.1080/0020739X.2016.1184337

\section{Document Version}

Author Accepted version

\section{General rights}

Copyright for the publications made accessible via Ulster University's Research Portal is retained by the author(s) and / or other copyright owners and it is a condition of accessing these publications that users recognise and abide by the legal requirements associated with these rights.

\section{Take down policy}

The Research Portal is Ulster University's institutional repository that provides access to Ulster's research outputs. Every effort has been made to ensure that content in the Research Portal does not infringe any person's rights, or applicable UK laws. If you discover content in the Research Portal that you believe breaches copyright or violates any law, please contact pure-support@ulster.ac.uk. 


\title{
Cannibalism and chaos in the classroom
}

\author{
Gavin M Abernethy \& Mark McCartney ${ }^{1}$ \\ School of Computing and Mathematics \\ University of Ulster
}

${ }^{1}$ m.mccartney@ulster.ac.uk 


\begin{abstract}
Two simple discrete-time models of mutation-induced cannibalism are introduced and investigated, one linear and one nonlinear. Both form the basis for possible classroom activities and independent investigative study. A range of classroom exercises are provided, along with suggestions for further investigations.
\end{abstract}




\section{Introduction}

The rapid growth in the study of complex and chaotic systems in applied mathematics over the last two decades has been driven and facilitated by the increased power of computers, and the availability of sophisticated packages such as MATLAB and Mathematica. While much of this work remains at the postgraduate and research level, simple discrete chaotic systems can be taught within the undergraduate curriculum, and provide good opportunities for students to develop skills in mathematical modelling, simple computer programming, and numerical investigation.

Common discrete time mathematical models met in undergraduate mathematics centre around mathematical ecology with the study of the logistic, Ricker and Beverton-Holt maps. ${ }^{1,2}$ In this paper we look at a variant of such single species population models, by considering a case where a species reproduces to produce mutants which are cannibals.

Perhaps surprisingly, cannibalistic behaviour does occur in nature and has been studied in fish populations $s^{3,4,5}$, salamanders ${ }^{6}$ and, more recently, in fruit fly larvae ${ }^{7}$ and tadpoles ${ }^{8}$. While cannibalistic behaviour is age/size structured in fish and salamanders, with adults consuming young in the group, this is not the case with fruit fly larva and tadpoles. In the research literature a range of discrete and continuous time models have been proposed, with Claessen $e t a l^{9}$ providing a useful overview of work done up to the early twenty-first century. Recent discrete time models have focussed on Ricker-like maps as a basis of population dynamics $^{10,11,12}$, however in this paper we will focus on a simple logistic equation based model which is more amenable to student investigation.

\section{A linear discrete-time model of cannibalism}

The simplest possible discrete time model of a population is the Malthusian equation

$$
x_{i+1}=r x_{i}
$$

where $x_{i}$ is the population at time step $i, r$ is the growth ratio and

$$
r=1+b-d
$$

where $d$ is the number of deaths and $b$ the number of births per head of population within the time-step. Clearly (1) can be solved to give

$$
x_{i}=r^{i} x_{0}
$$

resulting in divergent growth in population if $r>1$.

In what follows we will assume that $d=1$, i.e. that each population lasts for only one time step, and so the factor $r$ corresponds the number of births produced per head of population. If at each time step a fraction $p$ of the births are mutants who are cannibals which then consume members of the remaining population, this can be modelled via 


$$
\begin{aligned}
& x_{i+1}=(1-p) r x_{i}-c y_{i} \\
& y_{i+1}=p r x_{i}
\end{aligned}
$$

where $y_{i}$ is the size of the cannibal population at time step $i$ and each cannibal consumes $c$ of the original population. This model can be analysed in one of two ways, the first is to write (4) in matrix form as

$$
\underline{X}_{i+1}=A \underline{X}_{i}
$$

where $\underline{X}_{i}=\left[\begin{array}{c}x_{i} \\ y_{i}\end{array}\right], A=\left[\begin{array}{cc}(1-p) r & -c \\ p r & 0\end{array}\right]$ and the solution of (5) is given by

$$
\underline{X}_{i}=A^{i} \underline{X}_{0}
$$

Alternatively, (4) can be written as the second order linear homogeneous difference equation,

$$
x_{i+2}-(1-p) r x_{i+1}+\text { cprx }_{i}=0 .
$$

Such discrete equations can be solved in an analogous way to linear homogeneous differential equations via a trial solution of the form $x_{i}=\alpha R^{i}$, which substituting into (7) leads to a characteristic equation for $R$ of the form

$$
R^{2}-(1-p) r R+c p r=0
$$

which has roots

$$
R_{ \pm}=\frac{1}{2}\left(r(1-p) \pm \sqrt{r^{2}(1-p)^{2}-4 p c r}\right)
$$

giving a general solution of (7) the form

$$
x_{i}=\alpha R_{+}^{i}+\beta R_{-}^{i} .
$$

Clearly depending on the form of (9) the behaviour of (10) can be either convergent, divergent, monotonic, or oscillatory (see classroom exercises 4 for the special case of $R_{+}=1$ )

\section{A nonlinear discrete-time model of cannibalism}

A standard and more realistic alternative to the Malthusian population growth model given by

(1) is the logistic model

$$
X_{i+1}=r X_{i}\left(1-\frac{X_{i}}{K}\right)
$$


where $K$ is the carrying capacity of the environment, modelling the fact of finite resources. Thus for $X_{i} \ll K$ the population grows roughly as (1), but then as the population increases the second term in (11) damps population growth. By change of variable $x_{i}=X_{i} / K$, where $x_{i}$ is the population density, (11) becomes

$$
x_{i+1}=r x_{i}\left(1-x_{i}\right)
$$

which is the well-known logistic map. The logistic map exhibits a rich variety of behaviour for $r \in[0,4]$ and is an archetypal chaotic system. We can use the logistic map as a basis for a more complex cannibalism model given by

$$
\begin{aligned}
& x_{i+1}=(1-p) r x_{i}\left(1-x_{i}-y_{i}\right)-c y_{i} \\
& y_{i+1}=\operatorname{prx}_{i}\left(1-x_{i}-y_{i}\right)+r x_{i} y_{i} .
\end{aligned}
$$

This could be considered a modification of a model presented in Maynard Smith's textbook ${ }^{13}$ with the addition of mutation. In (13) the population $x$ mutates with probability $p$ to the cannibal population $y$, which then cannibalises the original population at a constant rate $c$. The $\left(1-x_{i}-y_{i}\right)$ term indicates that both populations share the same finite environment, while the term $r x_{i} y_{i}$ gives $y$ a simple Malthusian growth ratio $r x_{i}$. A standard approach to such models in a teaching environment is to attempt to solve (13) for fixed points and analyse their stability. However, for all but the simplest systems such analysis can prove unwieldy and it is much more useful and interesting to analyse the system numerically. The coding of a two dimensional map such as (13) is straightforward, making for only a few lines of code, and it is easy to check whether after a large number of iterations the system is diverging, or converging to a fixed point. To discover for what values of the parameter space the system is chaotic requires the evaluation of the global Lyapunov exponent, and this certainly requires numerical evaluation.

The evaluation of the global Lyapunov exponent for a one-dimensional map of the form

$$
x_{i+1}=f\left(x_{i}\right)
$$

is given by

$$
\lambda_{g}=\lim _{i \rightarrow \infty} \frac{1}{N} \sum_{i=1}^{N} \ln \left|f^{\prime}\left(x_{i}\right)\right|
$$

For any given map this is relatively straightforward to calculate numerically with the standard procedure being to iterate the map until transients have died out and then to estimate $\lambda_{g}$ using (15) over a large number of iterations, typically $\mathrm{N}=10^{6}$ or greater. $\lambda_{g}>0$ for non-divergent trajectories indicates chaos. The method of calculating the global Lyapunov exponent (or more accurately, because there are two, the largest Lyapunov exponent) for a two dimensional map is slightly more involved, but has been laid out by $\operatorname{Sprott}^{14}$ as follows: 
If we consider a map of the form

$$
\begin{aligned}
& x_{i+1}=F\left(x_{i}, y_{i}\right) \\
& y_{i+1}=G\left(x_{i}, y_{i}\right)
\end{aligned}
$$

and we have two points $\left(x_{i}, y_{i}\right),\left(x_{i}+\Delta x, y_{i}+\Delta y\right)$ separated by a distance $(\Delta x, \Delta y)$, then after one iteration they will be separated by $\left(\Delta x^{\prime}, \Delta y^{\prime}\right)$ where

$$
\begin{aligned}
& \Delta x^{\prime}=\frac{\partial F}{\partial x} \Delta x+\frac{\partial F}{\partial y} \Delta y \\
& \Delta y^{\prime}=\frac{\partial G}{\partial x} \Delta x+\frac{\partial G}{\partial y} \Delta y .
\end{aligned}
$$

The local Lyapunov exponent is defined as

$$
\lambda=\ln \left|\frac{\sqrt{\Delta x^{\prime 2}+\Delta y^{\prime 2}}}{\sqrt{\Delta x^{2}+\Delta y^{2}}}\right|
$$

with the global Lyapunov being the average Lyapunov exponent calculated over may iterations of the map. The global Lyapunov can thus be calculated via as

$$
\lambda_{g}=\lim _{N \rightarrow \infty} \frac{1}{2 N} \sum_{i=1}^{N} \ln \left[\frac{\left(\frac{\partial F_{i}}{\partial x}+\frac{\partial F_{i}}{\partial y} Y_{i}^{\prime}\right)^{2}+\left(\frac{\partial G_{i}}{\partial x}+\frac{\partial G_{i}}{\partial y} Y_{i}^{\prime}\right)^{2}}{1+Y_{i}^{\prime 2}}\right]
$$

where $\frac{\partial F_{i}}{\partial x}$ is the partial derivative of $F$ with respect to $x$ evaluated at $\left(x_{i}, y_{i}\right)$ etc., and

$$
Y_{i+1}^{\prime}=\frac{\frac{\partial G_{i}}{\partial x}+\frac{\partial G_{i}}{\partial y} Y_{i}^{\prime}}{\frac{\partial F_{i}}{\partial x}+\frac{\partial F_{i}}{\partial y} Y_{i}^{\prime}}
$$

is the tangent of the direction of maximum growth (or least contraction), and is independent of $Y_{0}^{\prime}$ after many iterations. Compared to the one-dimensional case (15) this may seem somewhat complicated, however the individual partial derivatives are easily calculated, and the resulting code is relatively short and is available on request from the authors. 


\section{Results}

The model described by (13) has three free parameters, $(p, r, c)$ and the initial condition $x_{0}$ to be set (we can reasonably assume that initially there are no mutants - i.e. $y_{0}=0$ ). Clearly $x_{0}, p \in[0,1]$ and in this paper we will consider $c, r \in[0,5]$. We will restrict our results to two indicative mutation rates of $p=10^{-3}$ and 0.1 , and the starting value $x_{0}=0.1$, and evaluate the global Lyapunov exponent for the map over $c, r \in[0,5]$. Results for starting values $x_{0}=0.3$ for $p=10^{-3}$ and 0.1 were also obtained, but are not presented here as, apart from some changes in the boundary at which ecological collapse occurs, they were broadly similar to the results obtained for $x_{0}=0.1$. For each choice of $\mathrm{c}$ and $r, 10^{5}$ iterations are performed to remove transients and then the global Lyapunov exponent was calculated over the next $10^{6}$ iterations.

We also present results for the average fraction of the population which is made up of mutant cannibals given by

$$
f=\frac{1}{n} \sum_{i=1}^{n} \frac{y_{i}}{x_{i}+y_{i}}
$$

where $10^{5}$ iterations are performed to remove transients before the average is taken of $n=10^{4}$ ratios.

Figure 1 summarises the results for (13). Figure 1(a) shows that for a mutation rate of $p=10^{-3}$ cannibals can coexist with non-mutants in the ecology up to and beyond cannibalism rates of $c=5$, whereas when the mutation rate increases to $p=0.1$ (Figure 1(b)) coexistence is not possible beyond approximately $c=3$. Figure $1(\mathrm{c})$, which shows the average fraction, $f$, of the population which is cannibalistic, reveals that for $p=10^{-3}$, and $r<2$ the proportion of cannibals is very small, and is only being populated by the first (mutation) term in (a), with the second term, representing the reproduction of the cannibals, being negligible. Comparing Figures 1(a) and 1(c) and 1(b) and 1(d) shows the broad trend that in the region of the parameter space where co-existence is possible, as the reproduction parameter $r$ increases and cannibalism rate $c$ decreases, the cannibal will occupy a greater fraction of the population, reaching a maximum of $f \simeq 0.5$ as $c$ approaches zero. This indicates that the ideal cannibal is one which has very restricted killing habits, and can survive by cannibalising infrequently.

Figures 1(a) and (b) also indicate that model can support periodic, quasi-periodic, and chaotic behaviour. Quasi-periodic behaviour is indicated by extended regions of $(c, r)$ space where $\lambda_{g}=0$. Details of a restricted region of $(c, r)$ space is shown in Figure 2 , for both $p=10^{-3}$ and $p=0.1$. Figure 2 illustrates the rich transitions in dynamic behaviour which can occur within this region of $(c, r)$ space, with bifurcations producing closed invariant curves (quasi- 
periodicity) and period-doubling cascades to chaos. An example of a strange attractor is shown in Figure 3, and Figure 4 gives an example of a quasi-periodic and a periodic attractor.

As is noted in Figure 1 extended regions where $\lambda_{\mathrm{g}}=0$ in $(c, r)$ space indicate quasi-periodic behaviour, whereas narrow ribbons or lines where $\lambda_{\mathrm{g}}=0$ are more likely to indicate bifurcations where the Lyapunov exponent rises to a local maximum at zero. This behaviour is illustrated in figure 5 .

\section{Conclusions and teaching use}

It may be mischievously suggested that one of the advantages of nonlinear discrete time systems in teaching is that they are not amenable to significant analytic investigation, and hence invite computational experimentation. This not only opens up the possibility of greater freedom in model creation, but also exposes the student to a wider range of tools and ideas that are basic to much research in applied mathematics, including simple computer programming, appropriate presentation of results, and the practical limitations of computation. Thus, for example, in the case of the results presented in Figures 1 and 2 it will not take students long to realise that moving from estimating the global Lyapunov exponent over $10^{6}$ iteration and $10^{7}$ iterations increases the runtime of the computer code by a factor of 10. If only one such Lyapunov exponent is to be calculated this is not significant, but when exponent has to be calculated for each combination of $(c, r)$ over a set of 1000x1000 grid points (as is the case in figures 1(a) and (b)) a factor of 10 increase in the run time can be very significant, and can lead the student to appreciate the importance both of efficient programming and pragmatic judgements about how 'accurate' is 'accurate enough'. Further, Figures 1 and 2 form a summary of not just a significant amount of computation, but also of a wide range of time series behaviours. One way for a student to appreciate this range is to plot a Feigenbaum diagram varying $r$ for a fixed $c$ (say $c=0.5$ ).

The work as presented in this paper is best suited to an independent undergraduate research project. However, it can also be utilised in a shorter time period in a classroom context. Presenting students with the model (13), Figures 1 and 2 and a description of their significance, they could be invited to investigate the behaviour of the map for individual combinations in $(c, r)$ space by plotting time series, or attractors in $(x, y)$ space, thus revealing for themselves the rich behaviours exhibited by the map.

Finally, the chaotic model described in this paper is only one of a class of models which can be constructed and investigated by students. For example, we have assumed that cannibals do not eat each other, which although possible could be generalised to allow indiscriminate cannibalistic behaviour. We have also assumed a somewhat ruthless efficiency of the cannibals, namely in any given population cycle they will consume at a constant rate $c$, irrespective of the density of non-mutant prey. Three possible alternative models are given in the classroom exercises below, however, students can be encouraged to devise and investigate others. 


\section{Classroom Exercises}

1. In the linear model of cannibalism set out in (4), do the mutants consume their peers, or the eggs/young of their peers?

2. It was noted in the text that the general solution of (5) could be given as (6). By evaluating the eigenvalues and eigenvectors of the matrix $A$ find the general solution of the system, and show that it is equivalent to (10).

3. What is the solution of (7) when the roots of the characteristic equation (8) are equal?

4. Given $r, c, p \geq 0$, and assuming $r^{2}(1-p)^{2}-4 p c r>0$, (9) implies that $R_{+}>R_{-}>0$. Show that when $r=(1-p(1+c)) \quad R_{+}=1$ and that for starting population $x_{0}$ and initially no cannibals,

$$
\lim _{i \rightarrow \infty} x_{i}=\frac{x_{0}}{1-R_{-}}
$$

5. Although in the text it is stated that the analysis of the fixed points of systems such as (13) can prove unwieldy, it is easy to show that this system has $(0,0)$ as a fixed point. By Calculating the eigenvalues of the associated Jacobian find for what parameter values this point is stable.

6. Figure 1(a) and (b) both show a clear lower boundary below which the population collapses. Show that this boundary is given by

$$
r=\frac{1}{1-p(1+c)}
$$

7. Figure 3 gives one example of a strange attractor for the map (13). By using figure 1 as a guide to which regions of $(c, r)$ give rise to chaotic behaviour plot other examples.

8. Plot a Feigenbaum diagram corresponding to Figure 5 to illustrate the range of behaviours of (13), $r \in[2.9,3.15]$ for a fixed value of $\mathrm{c}=0.6$ with $\mathrm{p}=10^{-3}, \mathrm{x}_{0}=0.1$.

9. Three possible alternatives to (13) are

$$
\begin{gathered}
x_{i+1}=(1-p) r x_{i}\left(1-x_{i}\right)-c y_{i} \\
y_{i+1}=\operatorname{prx}_{i}\left(1-x_{i}\right)+\frac{r}{2}\left(1+x_{i}\right) y_{i}\left(1-y_{i}\right) \\
x_{i+1}=(1-p) r x_{i}\left(1-x_{i}-y_{i}\right)-c x_{i} y_{i} \\
y_{i+1}=\operatorname{prx}_{i}\left(1-x_{i}-y_{i}\right)+r x_{i} y_{i} .
\end{gathered}
$$




$$
\begin{aligned}
& x_{i+1}=(1-p) r x_{i}\left(1-x_{i}-y_{i}\right)-c x_{i} y_{i} \\
& y_{i+1}=\operatorname{prx}_{i}\left(1-x_{i}-y_{i}\right)+r c y_{i} .
\end{aligned}
$$

How could such models be interpreted in terms of mutation-induced cannibalism? What are their strengths and weaknesses compared to the original model (13)? Pick one of the models and investigate its behaviour following the computational analysis laid out in the paper. 


\section{Figure Captions}

Figure 1 Behaviour of cannibalism model (13). In figures (a) $p=10^{-3}, x_{0}=0.1$, and (b) $p=0.1$, $x_{0}=0.1$ the largest Lyapunov exponent $\lambda_{g}$ is evaluated. In each case the map it iterated $10^{5}$ to remove transients before the Lyapunov exponent is calculated over a further $10^{6}$ iterations. Given we are estimating $\lambda_{g}$ via (19) with $N=10^{6}$ iterations, we place a $10^{-3}$ error bound on our calculations. Thus, black regions represent chaotic behaviour $\left(\lambda_{g}>10^{-3}\right)$; dark grey periodic orbits (period greater than one); light grey, period one orbits (both $\lambda_{g}<-10^{-3}$ ). In grey regions $-10^{-3}<\lambda_{g}<10^{-3}$. Extended regions of grey in $(c, r)$ indicate quasi-periodic behaviour, whereas narrow ribbons of grey are more likely to indicate bifurcations where the maximal Lyapunov exponent only touches zero (see Figure 5). In white regions the ecology has collapsed. In figures (c) $p=10^{-3}, x_{0}=0.1$, and (d) $p=0.1, x_{0}=0.1$, the fraction of the population which is made up of mutant cannibals as calculated by (21) is shown. In each case the map is iterated $10^{5}$ times to remove transients before the average is taken of $n=10^{4}$ ratios.

Figure 2 Details of largest Lyapunov exponents from Figure 1. (a) $p=10^{-3}, x_{0}=0.1$, (b) $p=0.1, x_{0}=0.1$.

Figure 3 Example of strange attractor for cannibalism model (13) (a) with $x_{0}=0.1, p=0.1$, $c=0.2, r=3.3$, (b) detail of a region of (a).

Figure 4 Periodic and quasi-periodic behaviour of model (13) (a) a period 7 orbit with $x_{0}=0.1, p=0.1, c=0.2, r=3.1$, (b) a quasi-periodic orbit with $x_{0}=0.1, p=0.1, c=0.2, r=3.05$.

Figure 5 Variation of the largest Lyapunov exponent. The behaviour of the largest Lyapunov exponent for $r \in[2.9,3.15]$ for a fixed value of $c=0.6$ with $p=10^{-3}, x_{0}=0.1$. The extended regions where $\lambda_{g}=0$ (e.g. between 2.9 and 3.0) indicate quasiperiodic behaviour, whereas the points where the Lyapunov exponent rises to local maxima at $\lambda_{g}=0$ (e.g. between 3.05 and 3.1) are indicative of bifurcations. 
Figure 1a

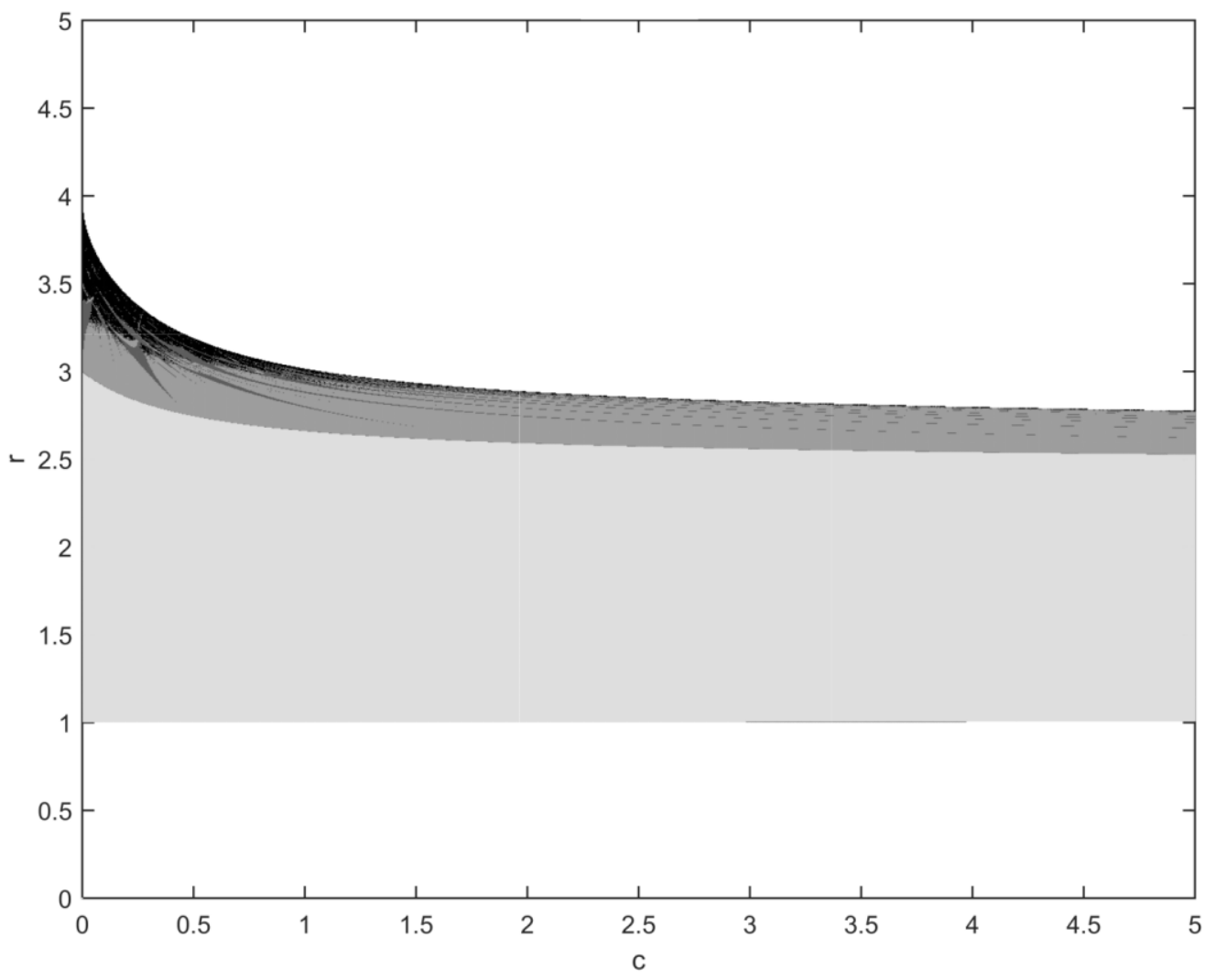

Figure 1b

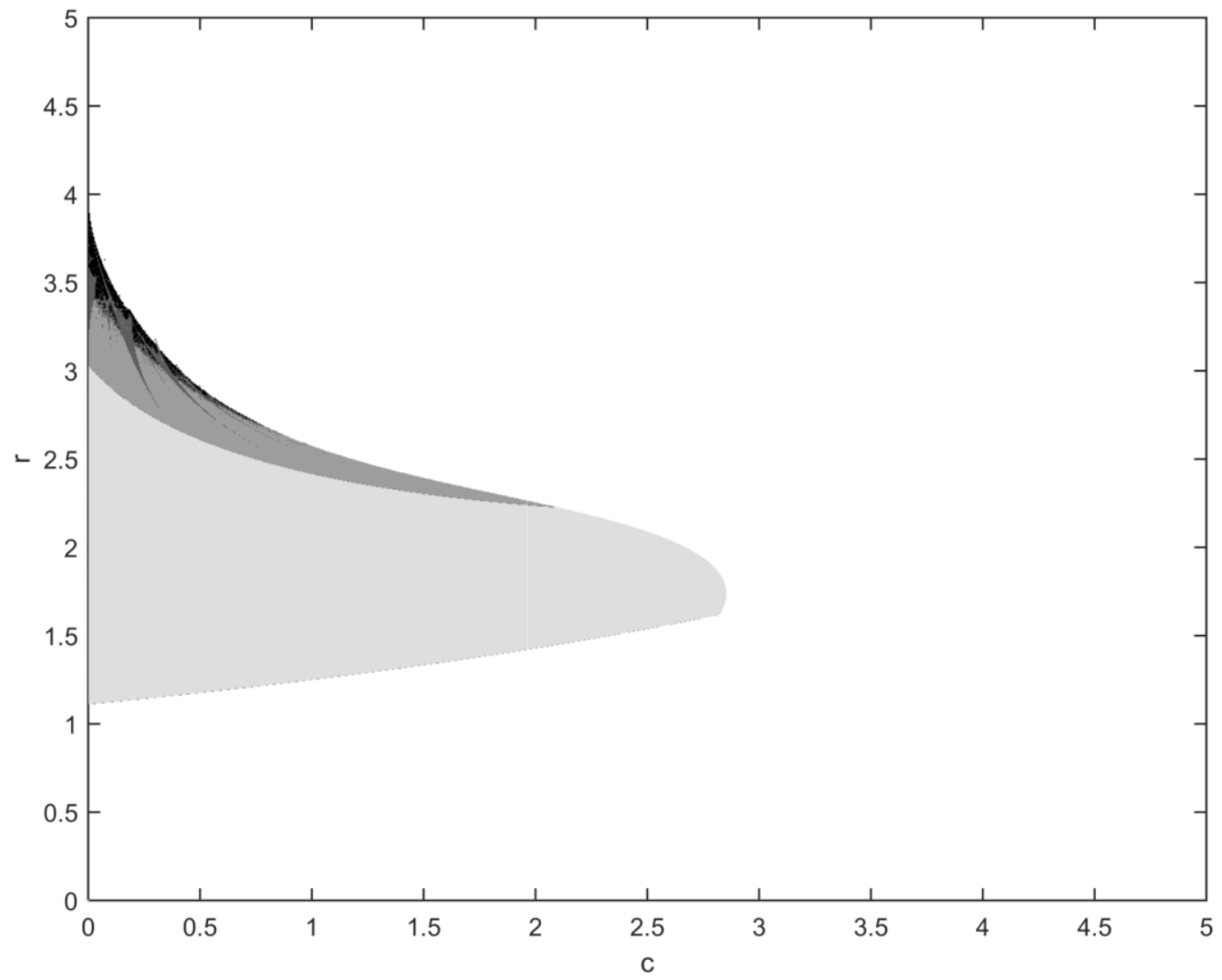


Figure 1c

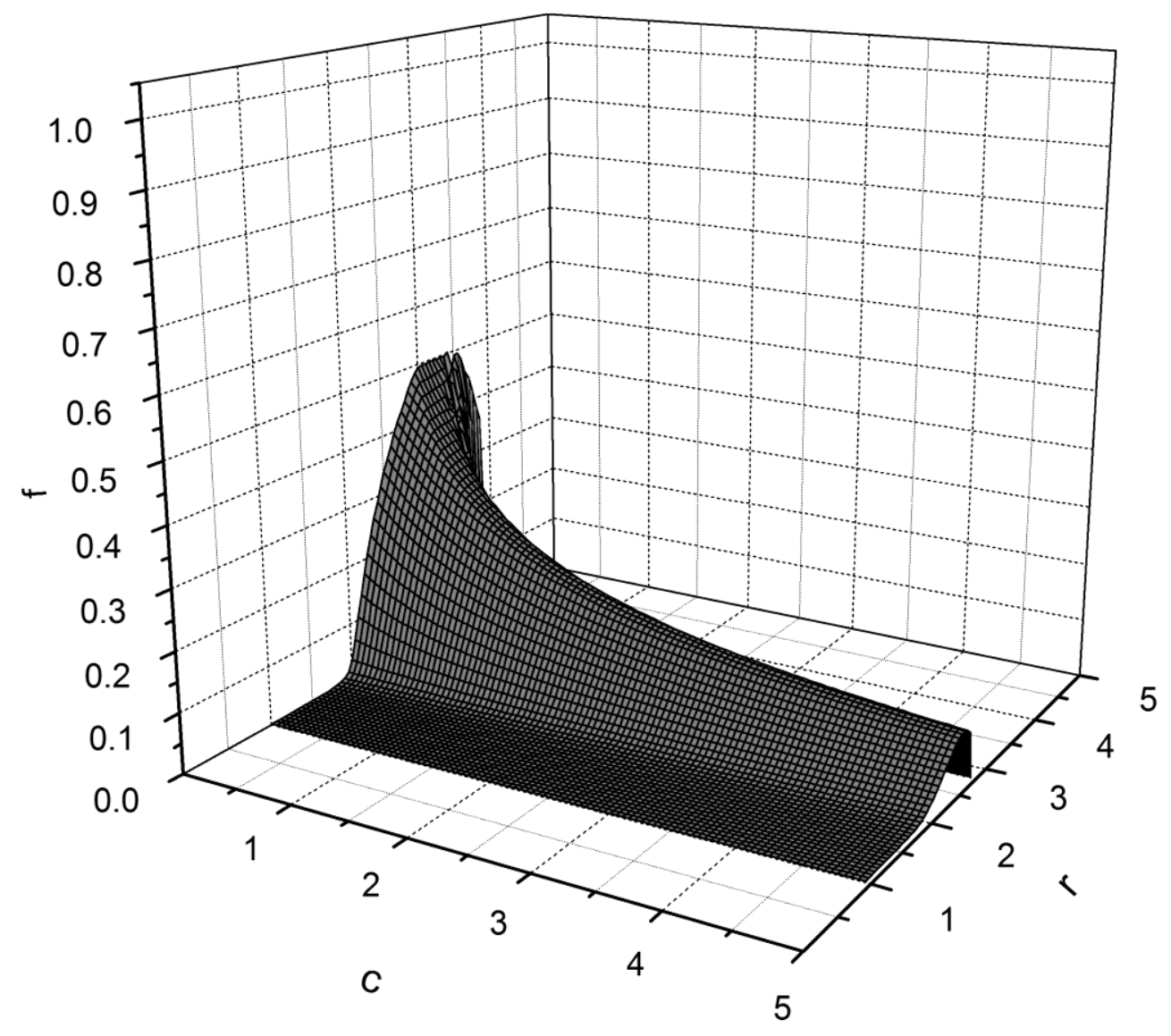


Figure 1d

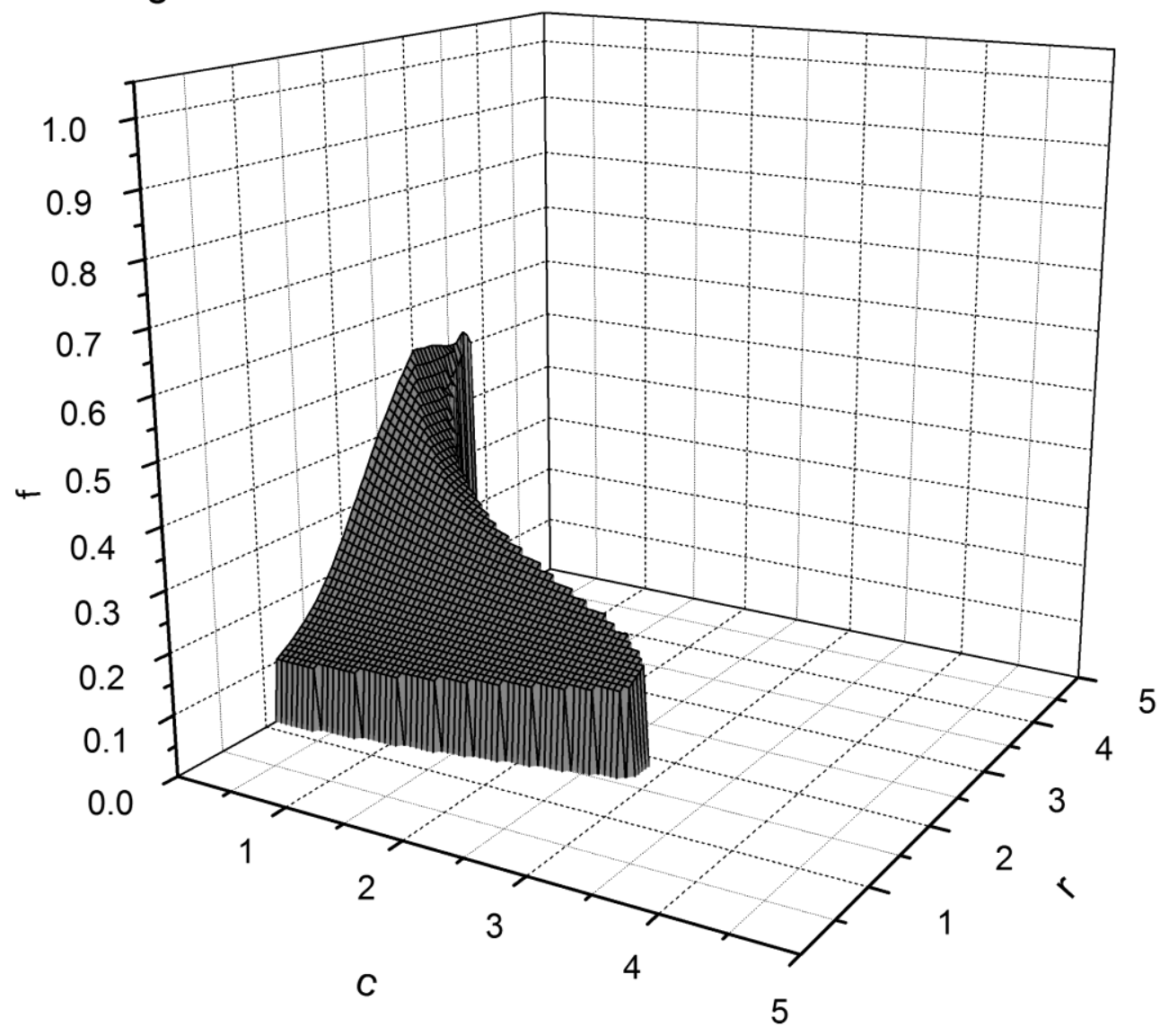


Figure 2a

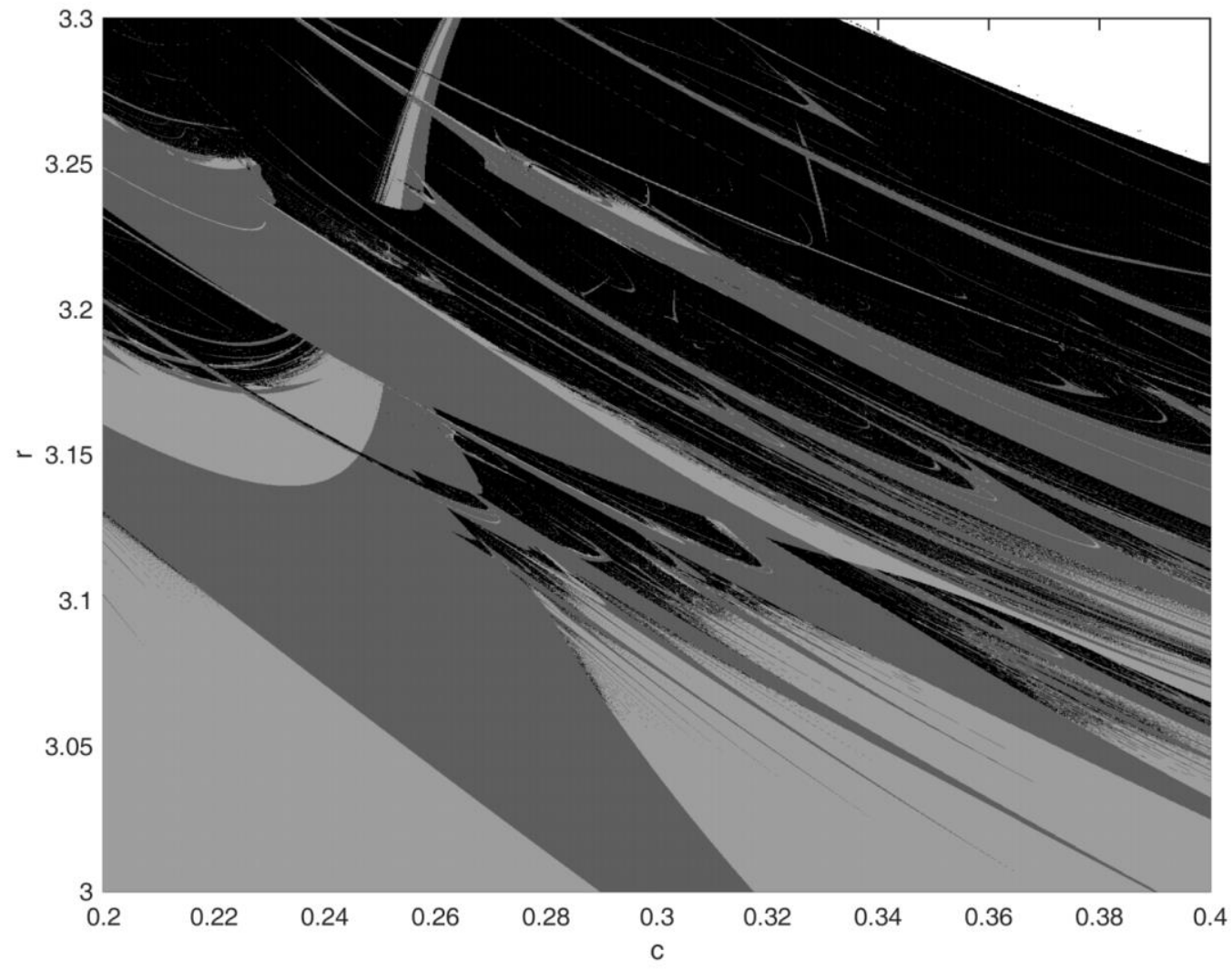


Figure $2 b$

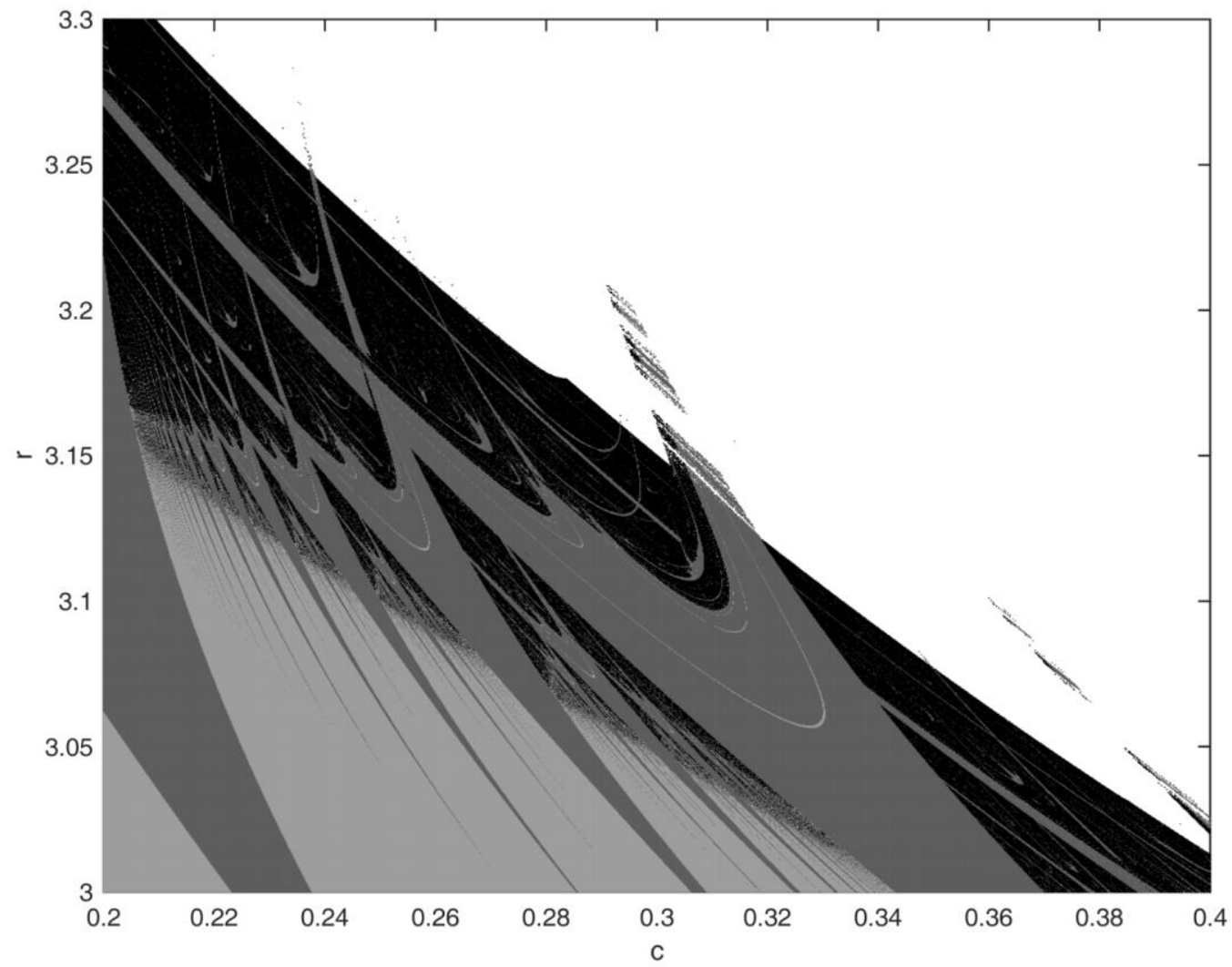




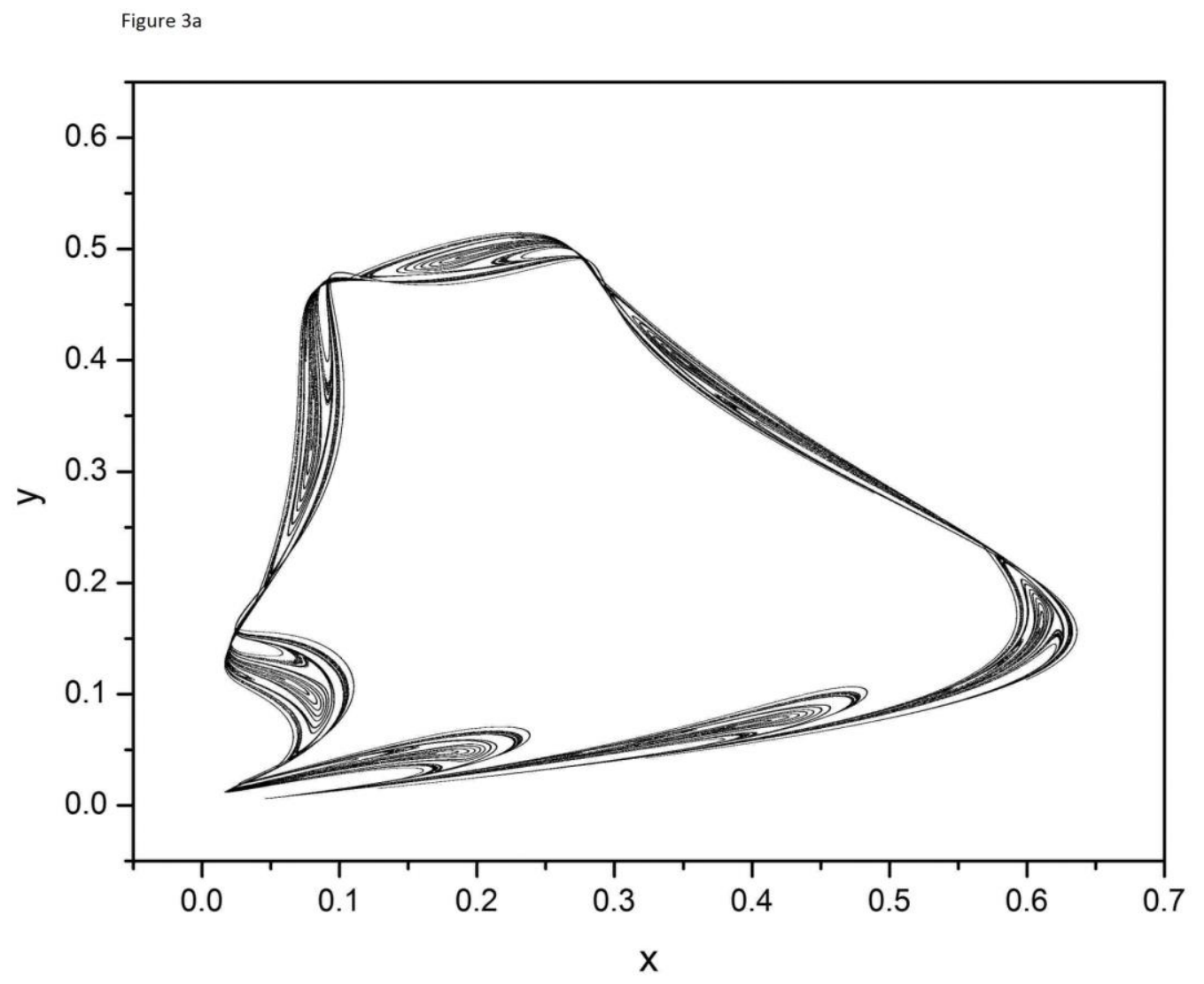




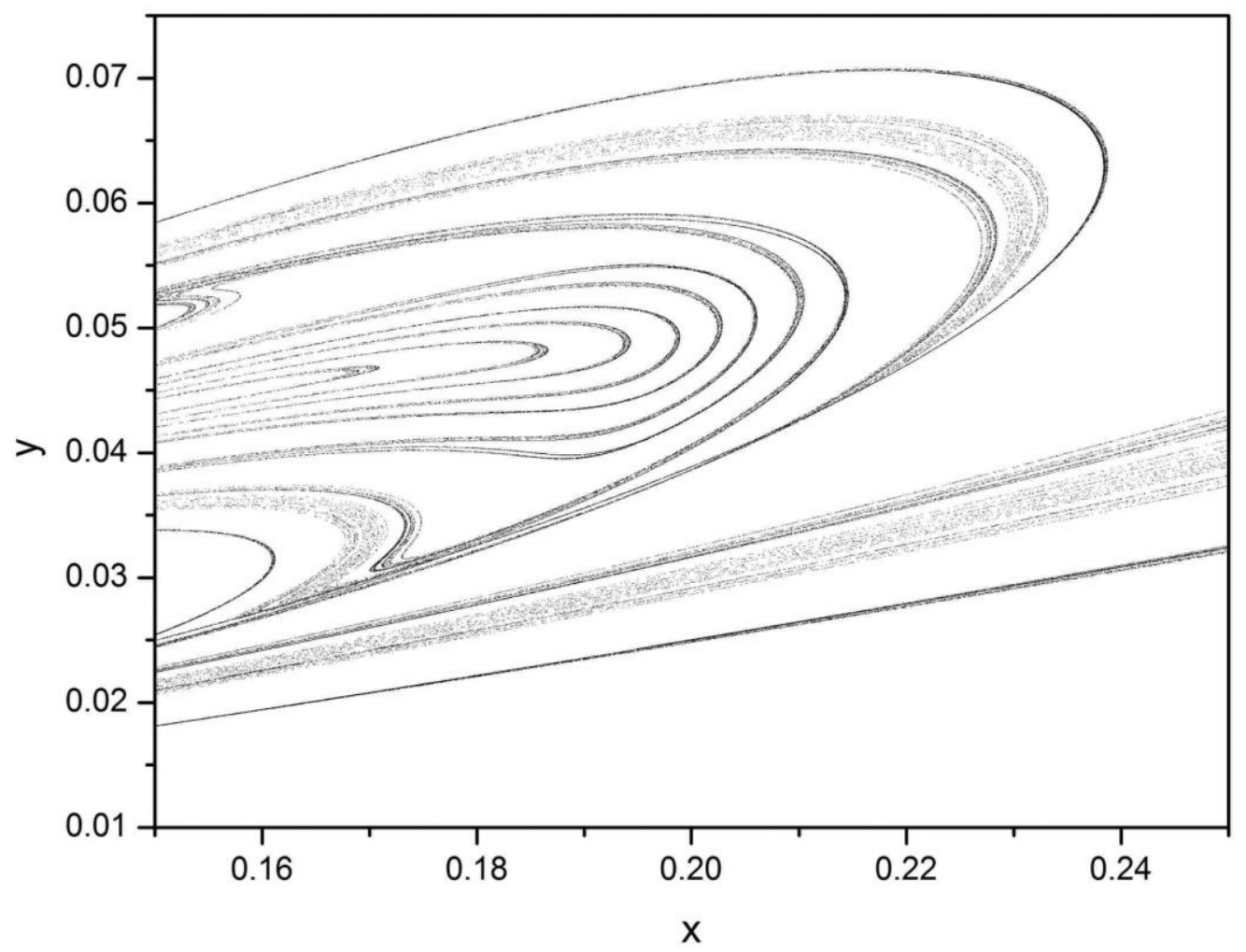


Figure 4 a

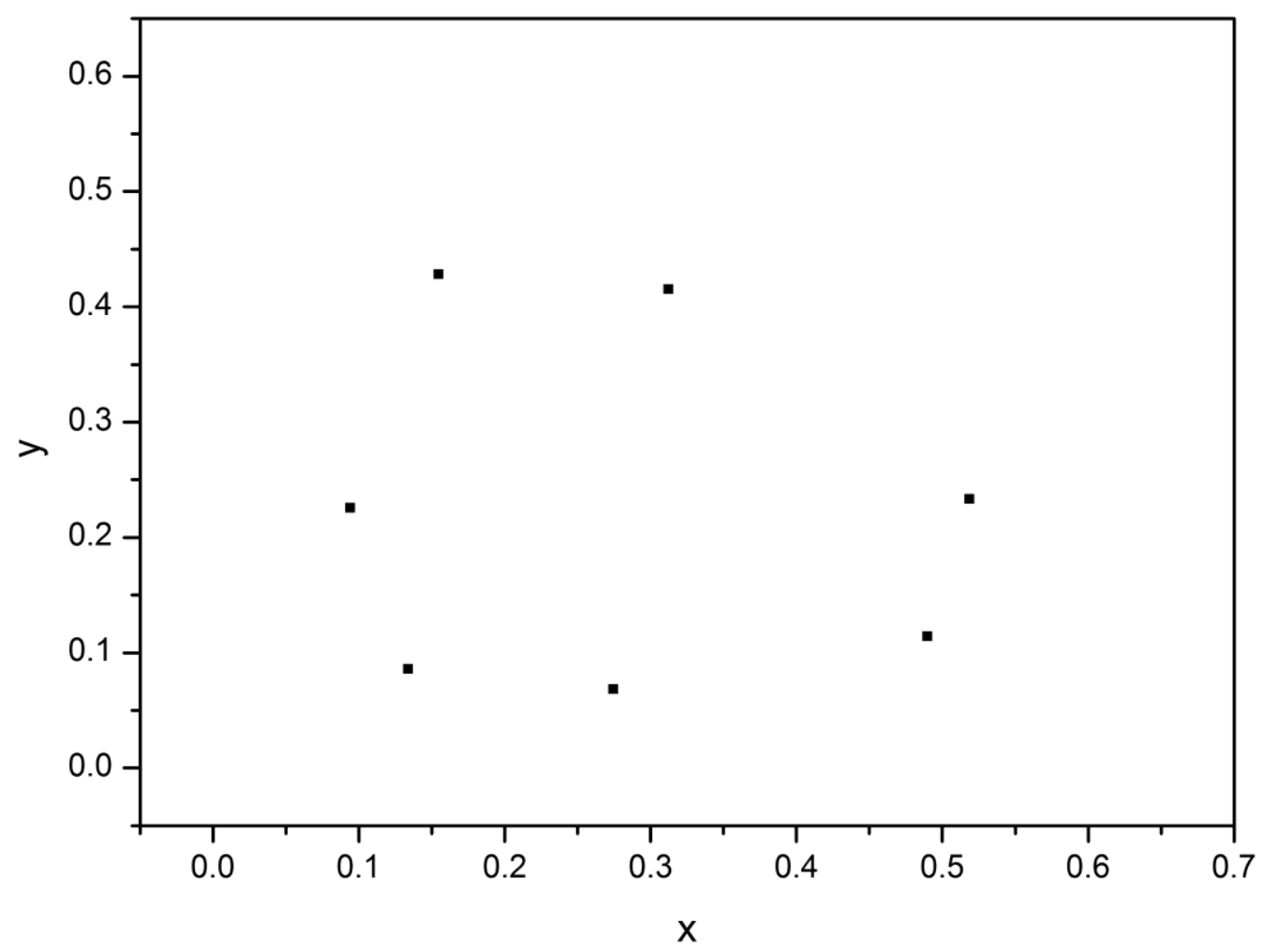


Figure $4 \mathrm{~b}$

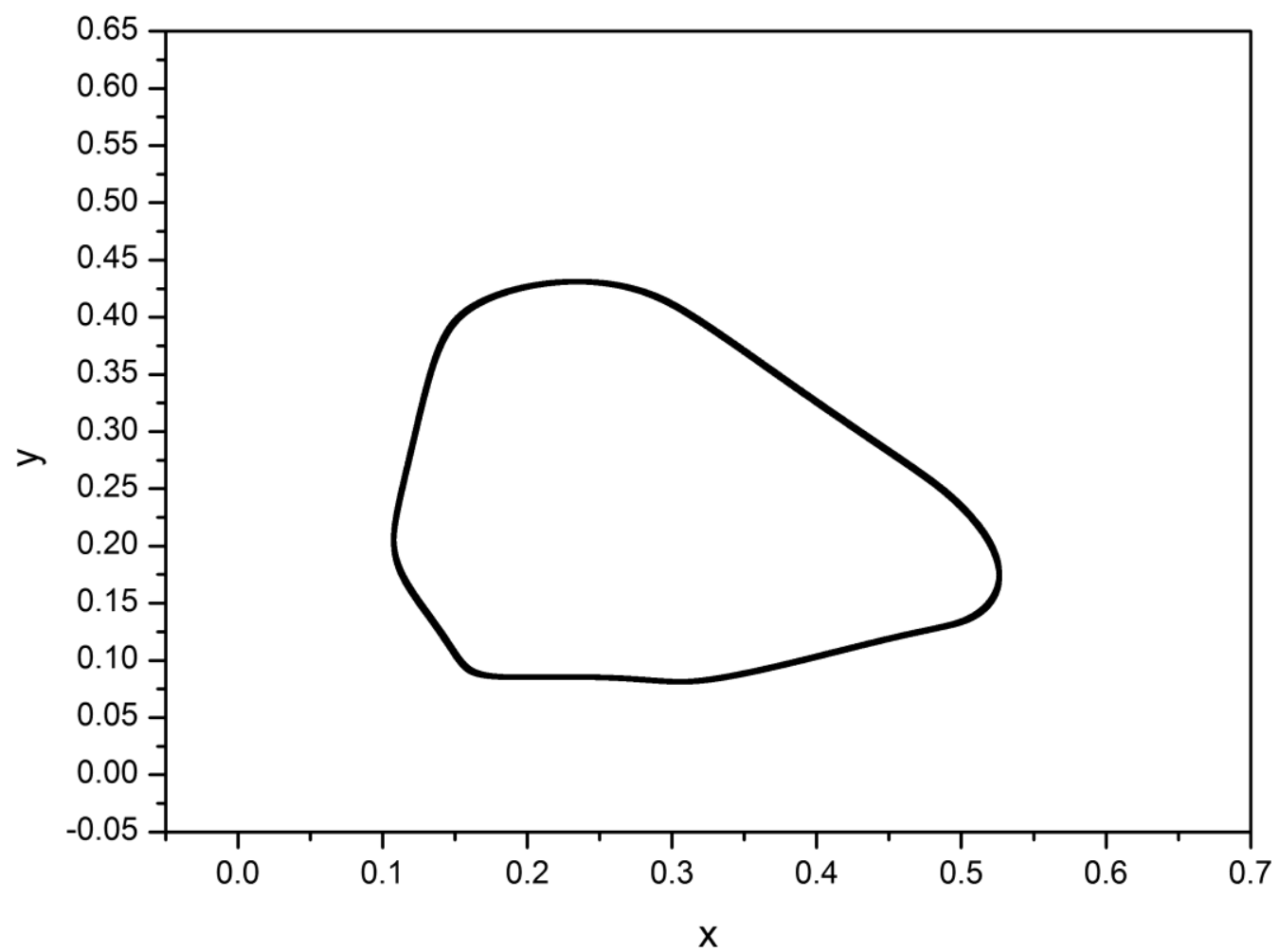


Figure 5

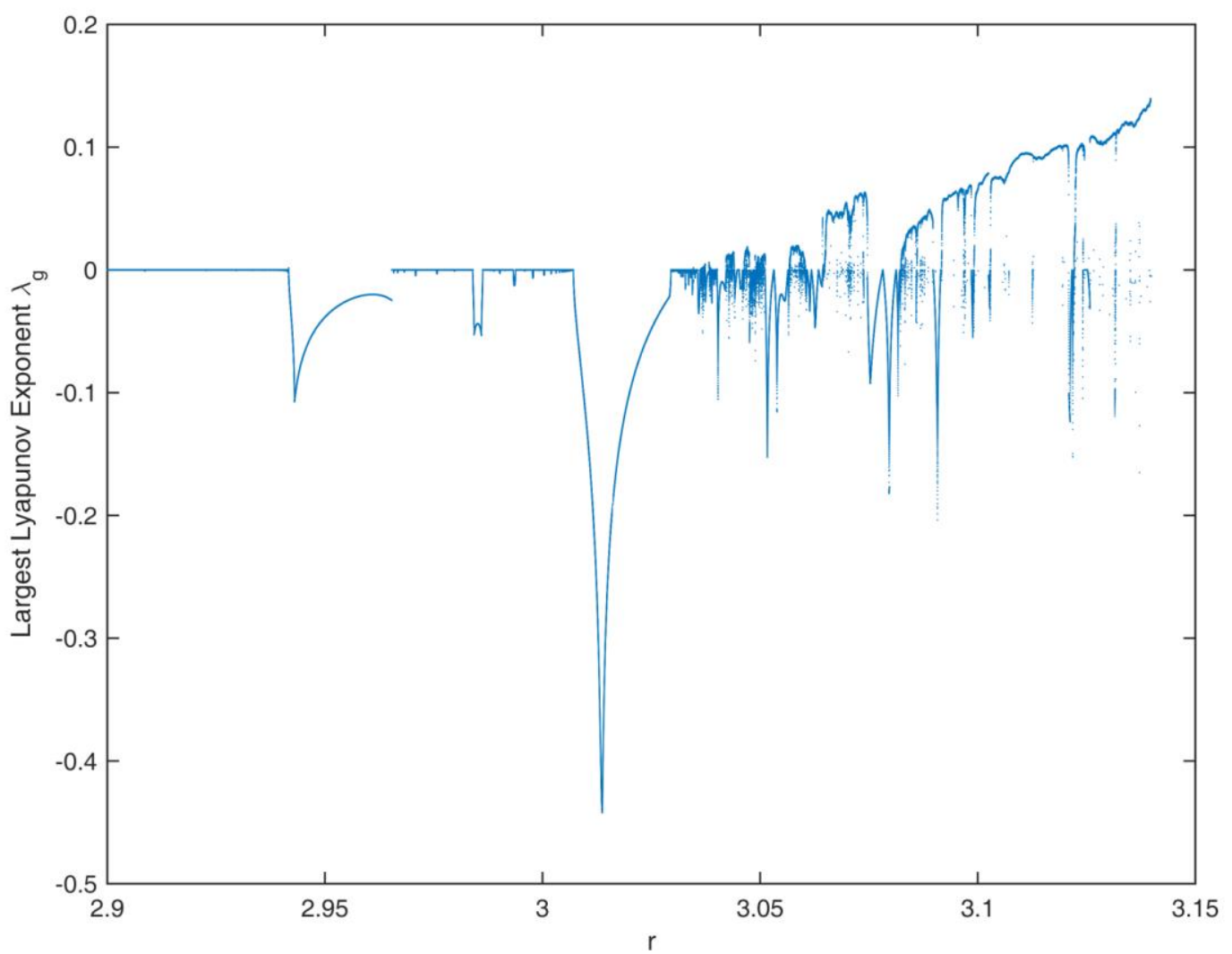




\section{References}

\footnotetext{
${ }^{1} \mathrm{~J}$. Banasiak, Mathematical modelling in one dimension, Cambridge University Press, 2013.

${ }^{2}$ Nicholas F. Britton, Essential mathematical biology, Springer-Verlag, 2005.

${ }^{3}$ L.R. Fox, Cannibalism in natural populations. Annual Review of Ecology and Systematics, 6, 87-106, 1975.

${ }^{4}$ B. Bogstad, G.R. Lili and S. Mehl, Cannibalism and year-class strength in Atlantic cod in Arcto-boreal ecosystems, ICES Marine Science Symposia 198 , 576-585, 1994.

${ }^{5}$ P. Byström, P. Ask, J. Andersson and L Persson, Preference for cannibalism and ontogenetic constraints in competitive ability of piscivorous top predators', Plos One 8(7), e70404, 2013.

${ }^{6}$ V.H.W. Rudolf, Impact of cannibalism on predator-prey dynamics: size-structured interactions and apparent mutualism, Ecology, 89(6), 1650-1660, 2008.

${ }^{7}$ R.K. Vijendravarma, S. Narasimha and T.J. Kawecki, Predatory cannibalism in Drosophila melanogaster larvae, Nature Communications, 4, 1789, 2013.

${ }^{8}$ D. M. Jefferson, K. A. Hobson, B. S. Demuth, M. C. O. Ferrari, D. P. Chivers, Frugal cannibals: how consuming conspecific tissues can provide conditional benefits to wood frog tadpoles (Lithobates sylvaticus), Naturwissenschaften, 101(4), 291-303, 2014.

${ }^{9}$ D. Claessen, A.M. de Roos and L Persson, Population dynamic theory of size-dependent cannibalism, Proceedings of the Royal Society B., 271, 333-340, 2004.

${ }^{10}$ S.R.-J. Jang and J-L Yu, Dynamics of a discrete-time host-parasitoid system with cannibalism, Journal of Biological Dynamics, 5(5), 419-435, 2011.

${ }^{11}$ Y. Chow and S. R.-J. Jang, Cannibalism in discrete-time predator-prey systems, Journal of Biological Dynamics, 6(1), 38-62, 2012.

${ }^{12}$ V.H.W. Rudolf, I. Sorrell and A.B. Pedersen, Revenge of the host: cannibalism, ontogenetic niche shifts, and the evolution of life-history strategies in host-parasitoid systems, Evolutionary Ecology Research, 14, 31-49, 2012.

${ }^{13}$ J. Maynard Smith. Mathematical Ideas in Biology, Cambridge University Press, 1968.

${ }^{14}$ J.C. Sprott, Chaos and time-series analysis, Oxford University Press, 2003.
} 